\title{
Effects of Academic Stress and Perceived Social Support on the Psychological Wellbeing of Adolescents in Ghana
}

\author{
Franklin N. Glozah \\ Department of Psychology and Human Development, Regent University College of Science and Technology, Accra, Ghana \\ Email: fglozah@hotmail.com
}

Received June 10, 2013; revised July 10, 2013; accepted July 18, 2013

Copyright (C) 2013 Franklin N. Glozah. This is an open access article distributed under the Creative Commons Attribution License, which permits unrestricted use, distribution, and reproduction in any medium, provided the original work is properly cited.

\begin{abstract}
The purpose of this study was to examine how academic stress and perceived social support influence the psychological wellbeing of Senior High School students in Ghana. Two hundred and twenty six male and female students participated. The general health questionnaire, student life-stress inventory and perceived social support from family and friends scales were used to assess psychological wellbeing, academic stress and perceived social support respectively. The results indicated that perceived social support buffered the effects of academic stress on psychological wellbeing. Girls reported higher scores on perceived social support but reported more depression. Boys reported higher academic stress and better psychological wellbeing, and these have been attributed partly to the socialisation role of gender. These results have policy implications in respect of the creation of a cordial school environment as well as encouraging a healthy interpersonal relationship between adolescents and their family and friends with the aim of reducing academic stress appraisal which is inimical to the psychological wellbeing of adolescents.
\end{abstract}

Keywords: Perceived Social Support; Academic Stress; Psychological Wellbeing; Adolescents

\section{Introduction}

Academic related stress coupled with poor interpersonal relationships with friends and family is a crucial factor in relation to the psychological wellbeing of adolescents. Frustrations and pressures to achieve in school, inter alia, are substantially mitigated by the perception of social support from significant others [1,2], which consequently has a positive impact on psychological wellbeing [3]. Long-term interpersonal difficulties such as simple misunderstandings may affect an individual's health and wellbeing by promoting a sense of hopelessness [4]. This phenomenon is particularly prevalent during adolescence when adolescents attain physical, sexual and social maturity and are influenced by little pressures and conditions such as academic stressors $[5,6]$. Adolescents become increasingly aware of the reciprocity involved in socially supportive relationships through social experiences during adolescence [7]. When adolescents are dissatisfied with their social support networks the likelihood of them developing anxiety, depression, or sleep problems increases substantially [8]. Academic stress coupled with low social support from family, friends, and others could have a negative impact on the health of students which will inevitably results in poor academic perform- ance [9]. Several studies have asserted that the mere fact of being in a supportive social network of family and friends could directly improve general health and wellbeing [10-12].

Stressful events mostly arise when an individual appraises a situation as threatening or challenging and does not have the appropriate coping skills to deal with it. These situations are ones in which the individual perceives that it is important to respond but an appropriate response is not immediately available [13]. Social support on the other hand basically refers to an individual's social contacts and network of relationships - it involves how an individual is integrated and influenced either directly or indirectly by another person $[14,15]$. Social support in this sense could be characterized as formal or informal relationships. Family, friends, colleagues at work or school and neighbors would be part of a person's informal set of relationships whiles doctors, lawyers and other professionals will be part of a person's formal set of social relationships. Each of these relationships, are enduring, providing encouragement, care, and direction to individuals, particularly to those in need.

A predominant characteristic of social support research has involved the theoretical testing of the "direct 
effect" and the "stress-buffering" hypotheses [16,17]. The fundamental idea behind the stress buffering hypothesis is that social support suppresses the deleterious effects of stress to promote or maintain good health [1821]. By implication the buffering hypothesis occurs only in the presence of stress, and is most useful to persons currently experiencing a high level of stressful events [22]. It has been posited that the stress buffering hypothesis becomes evident when supported and unsupported individuals differ significantly under low stress with even greater differences under high stress [23].

The direct or main effect hypothesis posits that positive perceptions of social support have a direct positive effect on health and wellbeing regardless or independent of stress $[24,25]$. The existence of an extensive, integrated network of social relations increases compliance to normative, health-promoting behaviours and provides sources of appropriate information and help [26]. Social support is a major coping resource in times of stress by mediating the effects of previous undesirable life events as well as suppressing the likelihood of subsequent life events. Social support both directly and indirectly affects depressive symptoms and significantly mediates the effects of undesirable life events [27]. Finch and Vega [12] hypothesised that social support will have a significant impact on individual-level physical health, net of sociodemographic characteristics. They found that discrimination and acculturation stress (i.e. stress developed as a result of coming into contact with a new culture) is relatively low for those who reported higher levels of social support and high among those who reported low social support.

It has also been asserted that the association between social support and psychological wellbeing differs between advantaged and disadvantaged groups, especially when it borders on material needs. [28] found that parental education was inversely related to adolescent substance use, with adolescents whose parents did not attain a higher level of education reporting lower levels for some protective factors and higher levels for some risk factors. Lower education attained was related to higher level of adolescent substance use, lower levels of protective factors, and higher levels of risk factors. This phenomenon has been corroborated and expressed to indicate that socio-economic status and the environment it creates for adolescence can influence the development of depression [5]. [29] supports this assertion by maintaining that economic difficulties can lead to parental stress and depression along with disrupted parental practices, which in turn affect the health and wellbeing of their children. Adolescents from lower socio-economic groups more frequently report low social support than those from high or middle socio-economic backgrounds in many studies [30].

\section{Objectives}

Specifically, the objectives of this study are:

1) To examine the buffering role of perceived social support in the association between stress and psychological wellbeing.

2) To examine the role of gender, age, socio-economic status and class level on psychological wellbeing.

3) To examine the role of gender, age, socio-economic status and class level on academic stress.

\section{Method}

\subsection{Research Design}

A questionnaire survey design was carried out among adolescents in Senior High Schools (SHS) in Accra, Ghana.

\subsection{Sample and Procedure}

The population for this study was Senior High School students in Accra, Ghana. Selecting the sample from this population was meant to ensure that the study has a well-represented sample that cuts across the whole country and for the results to possess a reasonably good level of external validity. In all, 248 questionnaires were distributed and out of this number 226 were returned. The 226 respondents consisted of $58 \%$ (131) males and $42 \%$ (95) females enrolled at a SHS which was randomly selected from a list of all SHSs in Accra. Respondents' ages ranged from 13 to 22 years $(M=17.07, S D=1.39)$.

To begin with, permission was sought from the school administration/authorities before the study could be carried out in the school, which was subsequently approved by the Regional Ethics Committee, Sør-Trondelag, Trondheim, Norway. Informed consent forms were given to each student to read and append their signatures if they agreed to willingly participate in the study. The questionnaires were given out randomly to all students per class/form (form 1, form 2, and form 3). Basically, every student in each class/form who consented to participate was given a questionnaire to complete. Those who agreed to participate and later changed their minds were asked to return the uncompleted questionnaires. Respondents were allowed to take as much time as they needed to complete the questionnaires.

\subsection{Measures}

\subsubsection{Student-Life Stress Inventory (SSI)}

The SSI by Gadzella [31] consists of 23 items representing five subscales of academic stressors i.e. frustrations, conflicts, pressures, changes, and self-imposition. Each items was on 5-point Likert scale response options from $1=$ never to $5=$ always, with higher scores indication 
more academic stress. [31] reported a Cronbach's alpha of 0.76 for the total SSI. A principal component analysis with varimax rotation confirmed that the SSI had five dimensions. Cronbach's alpha in the present study was $0.76,0.62,0.71,0.75$, and 0.84 for the frustrations, conflicts, pressures, changes, and self-imposition dimensions respectively.

\subsubsection{General Health Questionnaire (GHQ)}

The 20-item version of the GHQ by Goldberg [32] was used to assess psychological wellbeing. Respondents indicated agreement or disagreement along a 4-point scale for each statement from 1 - better than usual to 4 much more than usual, with higher scores indicating better psychological wellbeing. The 20 -item version had a reliability of $\alpha=0.84$ in the present study. When testretest reliability for the GHQ was conducted Goldberg [32] found a reliability of 0.90 . A principal component analysis with varimax rotation showed that the GHQ has six dimensions: depression $(\alpha=0.82)$, anxiety $(\alpha=0.71)$, social dysfunction $(\alpha=0.73)$, self-esteem $(\alpha=0.52)$, social phobia $(\alpha=0.59)$, and ability to concentrate $(\alpha=$ $0.61)$.

\subsubsection{Perceived Social Support from Family (PSS-FA) and Friends (PSS-FR) Scale}

The 10-item version each for social support from family (PSS-FA) and friends (PSS-FR) scale by Procidano and Heller [33] was used. Each subscale scores range from 1 to 10 with higher scores indicating more social support. According to Procidano and Heller [33] the PSS-FA and PSS-FR is a homogeneous measure with Cronbach's $\alpha$ of 0.88 and 0.90 respectively. In the present study the Cronbach's $\alpha$ of the total PSS was $\alpha=0.82$ with PSS-FA $\alpha=814$ and PSS-FR $\alpha=0.75$.

\subsubsection{Measures of Socio-Economic Status (SES)}

To determine the socio-economic status of students, their parent's highest education attained was used. Respondents were asked to indicate the highest level of education attained by either parent. Educational level was classified as: 1) university doctorate (high socio-economic status), 2) university master's degree (high socio-economic status), 3) university bachelor's degree (middle class), 4) form four/secondary school (low socio-economic status), and 5) no education (low socio-economic status).

\subsection{Statistical Analysis}

A two-way analysis of covariance (ANCOVA) was conducted to examine the main and interaction (buffering) effects of PSS (high, moderate, and low) and SSI (low, moderate, and high) on the GHQ. A partial eta squared $\left(\eta_{\mathrm{p}}^{2}\right)$ was used to indicate the effect size indices of the main and interaction effects of PSS and SSI. A multivariate analysis of variance (MANOVA) was applied to examine between-subject effects of the independent variables of gender, age, class level, and socio-economic status on GHQ (depression, anxiety, social dysfunction, social phobia, and self-esteem). Another multivariate analysis of variance (MANOVA) was applied to examine between-subject effects of the independent variables of gender, age, class level, and socio-economic status on the SSI (frustrations, pressures, conflicts, changes, and selfimposition). Further, one-way or two-way analyses of variance (ANOVA) and t-test were applied to examine specific group differences in case any significant difference or variances were found. Data analysis was conducted using the Statistical Package for Social Science (SPSS 15) software.

\section{Results}

\subsection{The Buffering Role of Perceived Social Support in the Association between Stress and Psychological Wellbeing}

It was found that the main effect of perceived social support was significant, indicating that psychological wellbeing is significantly higher for students who reported high perceived social support than those who reported moderate and low perceived support, with a small effect size. The main effect of academic stress was also significant indicating that psychological wellbeing was significantly higher for students who reported moderate academic stress than those who reported high and mild academic stress, with a large effect size. Level of perceived social support had a higher significance level and a larger effect size than level of academic stress, indicating that academic stress differences in psychological wellbeing is influenced by the level of perceived social support. Table 1 shows the means and standard deviation of levels of perceived social support and academic stress and Table 2 shows the two-way ANCOVA of social support and academic stress on psychological wellbeing.

\subsection{Effects of Gender, Age, Socio-Economic Status and Class Level on Psychological Wellbeing}

From Table 3 it can be observed from the MANOVA results that the overall effect of age, class level, gender and socio-economic status on psychological wellbeing was not significant. However, there were gender differences in psychological wellbeing and these were recorded on depression $(F=7.58, p<0.05)$ and social dysfunction $(F=6.31, p<0.05)$. A further independent samples t-test with gender as the independent variable and 
Table 1. Means and standard deviations of levels of social support and levels of academic stress on psychological wellbeing.

\begin{tabular}{|c|c|c|c|}
\hline $\begin{array}{c}\text { Level of } \\
\text { Academic Stress }\end{array}$ & $\begin{array}{l}\text { Level of Perceived } \\
\text { Social Support }\end{array}$ & Mean & SD \\
\hline \multirow[t]{4}{*}{ Low academic stress } & Low support & 27.24 & 12.55 \\
\hline & Moderate support & 24.55 & 7.03 \\
\hline & High support & 26.07 & 16.94 \\
\hline & Total & 25.82 & 11.58 \\
\hline \multirow[t]{4}{*}{ Moderate academic stress } & Low support & 29.91 & 11.30 \\
\hline & Moderate support & 27.29 & 7.14 \\
\hline & High support & 18.85 & 8.07 \\
\hline & Total & 25.52 & 10.09 \\
\hline \multirow[t]{4}{*}{ High academic stress } & Low support & 39.77 & 13.50 \\
\hline & Moderate support & 26.63 & 8.03 \\
\hline & High support & 24.25 & 11.90 \\
\hline & Total & 31.23 & 13.21 \\
\hline \multirow[t]{4}{*}{ Total } & Low support & 32.51 & 13.56 \\
\hline & Moderate support & 25.96 & 7.39 \\
\hline & High support & 22.67 & 12.56 \\
\hline & Total & 27.51 & 11.95 \\
\hline
\end{tabular}

Table 2. Two-way ANCOVA of levels of social support and levels of academic stress on psychological wellbeing with age, gender, ses, and class level as covariates.

\begin{tabular}{ccccccc}
\hline & $\begin{array}{c}\text { Sum of } \\
\text { Squares }\end{array}$ & $d f$ & $\begin{array}{c}\text { Mean } \\
\text { Square }\end{array}$ & $F$ & $p$ & $\eta_{\mathrm{p}}^{2}$ \\
\hline Social Support & 2770.25 & 2 & 1385.13 & 11.98 & $0.000^{* * *}$ & 0.11 \\
Academic stress & 1009.67 & 2 & 504.83 & 4.37 & $0.014^{*}$ & 0.05 \\
$\begin{array}{c}\text { Social support x } \\
\text { Academic Stress }\end{array}$ & 1599.41 & 4 & 399.85 & 3.46 & $0.009^{* *}$ & 0.07 \\
${ }^{*} p<0.05,{ }^{* *} p<0.01,{ }^{* * *} p<0.001$. & & & & \\
\hline
\end{tabular}

Table 3. MANOVA of Dimensions of psychological wellbeing by age, class level, gender and socio-economic status.

\begin{tabular}{|c|c|c|c|c|}
\hline & Wilks' $\lambda$ & $F$ & $d f$ & $p$ \\
\hline Age & 0.92 & 0.94 & 4 & 0.59 \\
\hline Class level & 0.93 & 0.76 & 12 & 0.70 \\
\hline Gender & 0.70 & 2.45 & 6 & $0.03 *$ \\
\hline Socio-economic status & 0.67 & 2.03 & 12 & $0.02 *$ \\
\hline Gender $^{*}$ Age ${ }^{*}$ Class ${ }^{*}$ SES & 0.95 & 0.62 & 12 & 0.83 \\
\hline
\end{tabular}

depression as dependent variable revealed a significant effect $t(198)=3.44, p<0.01$ with females $(M=10.72$, $S D=4.43)$ experiencing more depression than males $(M$ $=8.57, S D=4.35)$. Another t-test with gender as independent variable and social dysfunction as dependent variable showed that males functioned better socially ( $M$ $=6.88, S D=2.48)$ than females $(M=7.69, S D=2.42)$, $t(198)=2.11, p<0.05$.

Socio-economic status was also found to have a significant effect on psychological wellbeing with the significant effects evident on anxiety $(F=5.88, p<0.05)$ and social phobia $(F=3.55, p<0.05)$. A one-way ANOVA showed a significant effect of socio-economic status on anxiety $F(2,199)=7.29, p<0.01$. Post hoc analyses using LSD showed that average anxiety was significantly lower among the high socio-economic status group $(M=3.19)$ than those in the middle class $(M$ $=3.77)$ and low socio-economic status group $(M=4.78)$. This indicates that students from high socio-economic backgrounds reported less anxiety than those in the middle class and low socio-economic class.

Another ANOVA test showed a significant effect of socio-economic status on social phobia $F(2,199)=3.22$, $p<0.05$. LSD comparisons revealed that average social phobia was significantly lower among the high socioeconomic status group $(M=2.88)$ than those in the middle class $(M=2.90)$ and low socio-economic status $(M=$ 3.53). This implies that those in the high socio-economic group reported less social phobia than those in the middle class and low socio-economic class.

\subsection{Effects of Gender, Age, Socio-Economic Status and Class Level on Academic Stress}

A MANOVA was performed with academic stress (frustrations, pressures, conflicts, changes, and self-imposition) as dependent variable and age, class level, gender and socio-economic status as independent variables. An overall near significant effect was found indicating that age, class level, gender, and socio-economic status do not cumulatively affect academic stress. Nonetheless, gender significantly affected academic stress and these were recorded on academic frustrations $(F=9.80, p<0.01)$ and self-impositions $(F=6.57, p<0.05)$. A further independent samples t-test examining gender differences in academic frustrations revealed a significant effect $t(198)$ $=3.31, p<0.01$ with males $(M=19.29, S D=4.73) \mathrm{re}$ porting more academic frustrations than females $(M=$ $16.66, S D=6.49)$. Another t-test with gender and selfimposition showed that females reported less self-imposition $(M=17.13, S D=5.69)$ than males $(M=19.39, S D$ $=3.52), t(198)=3.47, p<0.01$.

Socio-economic status was also found to have a significant effect on academic stress with the significant 
effects evident on academic frustrations $(F=12.42, p<$ $0.001)$, self-imposition $(F=13.61, p<0.001)$, and academic pressures $(F=4.56, p<0.05)$. A further ANOVA showed a significant effect of socio-economic status on academic frustrations $F(2,199)=18.70, p<0.000$. Post hoc LSD analyses indicated that average academic frustrations was significantly lower among the high socioeconomic status group $(M=12.97)$ than those in the middle class $(M=18.97)$ and low socio-economic status group $(M=19.19)$. This indicates that students from high socio-economic backgrounds reported less academic frustrations than those in the middle and low socio-economic classes. Another ANOVA test showed a significant effect of socio-economic status on self-imposition $F(2,199)=$ 30.69, $p<0.000$. LSD comparisons revealed that the mean of self-imposition was significantly lower among the high socio-economic status group $(M=13.44)$ than those in the middle $(M=17.74)$ and low socio-economic status groups $(M=19.74)$. This implies that those in the high socio-economic group reported less self-imposition than those in the middle class and those from low socioeconomic backgrounds.

Finally, a one-way ANOVA revealed a significant difference between high, middle and low socio-economic status in academic pressures $F(2,199)=6.90, p<0.01$. Post hoc LSD analyses indicated that average academic pressures was significantly lower among the high socioeconomic status group $(M=7.84)$ than those in the low socio-economic status group $(M=10.15)$ and the middle class $(M=10.35)$. This indicates that students from high socio-economic backgrounds reported less academic pressures than those in the middle class and low socioeconomic background but those in the low socio-economic status group reported lesser academic pressures than those in the middle class. Age and class level did not have a significant effect on academic stress. Table 4 below shows the MANOVA results for Age, class level, gender and socio economic status on academic stress.

\section{Discussion}

The aim of this study was to examine the effects of per-

Table 4. MANOVA of dimensions of academic stress by age, class level, gender and socio-economic status.

\begin{tabular}{|c|c|c|c|c|}
\hline & Wilks' $\lambda$ & $F$ & $d f$ & $p$ \\
\hline Age & 0.97 & 0.83 & 4 & 0.76 \\
\hline Class level & 0.92 & 1.07 & 12 & 0.39 \\
\hline Gender & 0.71 & 2.50 & 5 & $0.03^{*}$ \\
\hline Socio-economic status & 0.52 & 4.55 & 10 & $0.000^{* * *}$ \\
\hline Gender $^{*}$ Age ${ }^{*}$ Class ${ }^{*}$ SES & 0.88 & 1.79 & 10 & 0.06 \\
\hline
\end{tabular}

${ }^{*} p<0.05,{ }^{*} p<0.01,{ }^{* * *} p<0.001$. ceived social support and academic stress on the psychological wellbeing of adolescents in Ghana.

The first objective was to examine the significance of the interaction effect between perceived social support and academic stress on psychological wellbeing. A significant interaction effect was found indicating that the impact of academic stress on psychological wellbeing depends substantially on perceived social support from family and friends. This finding is consistent with the stress-buffering hypothesis that perceived support protects individuals from life stress which in turn enhances psychological wellbeing $[18,19,21,34]$. It however runs contrary to studies in which the buffering hypothesis was not supported [35,36].

As an interpretation of this finding, it has been emphasized that students who experience low academic stress are likely to have a higher self-esteem and self-confidence [37,38], and so are more likely to seek support from family and friends whenever they are in need. As incorporated in the stress buffering hypothesis, perceived social support may ease the impact of stress appraisal by providing a solution to a problem, by reducing the perceived importance of the problem, or by neutralizing the neuroendocrine system so that people are less reactive to perceived stress, or by facilitating healthy behaviors [16]. The finding could also mean that students who reported high academic stress may have subsequently developed low self-esteem and self-confidence which is pivotal in an individual's sense of psychological wellbeing. According to Thoits [39] coping and social support eliminates or alters problematic demands, or they control the feelings of anxiety or depression usually engendered by those demands". The central tenet of the buffering hypothesis is that the presence of social support will decrease the likelihood of the development of psychological symptoms in the presence of stress [40].

The second objective of this study was to examine the overall effect of age, class level, gender, and socio-economic status on psychological wellbeing. A significant overall effect was not found indicating that psychological wellbeing does not depend on the combined effects of age, class level, gender, and socio-economic status. However, the results clearly pointed out that psychological wellbeing varies depending on gender and socio-economic status. Specifically, there were gender differences in depression and social dysfunction whereas socioeconomic status recorded differences in anxiety and social phobia. Consistent with previous findings the results showed that girls had higher depression and social dysfunction levels than boys e.g. [41]. This however did not support findings of some previous studies e.g. [42]. The gender differences in depression and social dysfunction could be interpreted in terms of gender role socialisation. Males in African cultures are nurtured to be active, asser- 
tive, outspoken, and to always fight for their freedom and independence whiles females are nurtured to be caring, passive, delicate, and always "sit and watch". As a result, boys are more likely to go about their daily life activities (i.e. social functioning) with ease and are less likely to develop depression compared to girls. Furthermore, males are culturally socialized to consider complaining as a weak attitude and "unmasculine", females on the other hand are socialized, perhaps biologically, to complain about the slightest problems they encounter (e.g. depression). Consequently, males may not have reported all or any problems they encounter either at home or at school.

Considering the fact that there were differences in social support in respect of socio-economic status, it can be speculated that low perceived social support by students from low socio-economic backgrounds would mean that they could not overcome problems which could have been solved with the help of their family and friends. Such parents, due to their socio-economic circumstances, are always concentrating on how to survive by means of working or seeking work, thereby paying little or no attention to the psychological wellbeing of their children. Conversely, the findings showed that students from high socio-economic backgrounds reported less anxiety and social phobia and this could be due to the fact that they reported more perceived social support than those in the middle and low socio-economic classes. As the educational level of parents was used as proxy for socio-economic status, it can be logically presumed that parents with higher education are likely to offer more support to their children. Parents who have had more education and thus better jobs and incomes are able to support their children and diverse ways. Interestingly, the age and class level of students did not have any significant effect on any category of their psychological wellbeing. This is consistent with the findings of previous studies $[43,44]$. The inclusion of age and class level might have accounted for the overall non significance. It has been asserted that there is no developmental basis for perceived social support, which has a significant effect on psychological wellbeing [45]. Moreover, age and class/form in school are theoretically synonymous, and the inclusion of both in the analysis may have been extraneous, hence the overall non significance observed.

The third objective of this study was to examine age, class level, gender, and socio-economic status differences in academic stress. There was no overall significant effect on academic stress indicating that academic stress does not depend on the combined effects of their age, class level, gender, and socio-economic status. Notwithstanding, the results pointed to the fact that there were academic stress differences in socio-economic status and gender. Particularly, gender had significant effects on academic frustrations and self-imposition while socio-economic status recorded significant effects on academic frustrations, self-imposition, and academic pressures. This finding is consistent with previous findings that being in a high socio-economic class reduces the level of academic stressors experienced [46]. Boys scored high on academic frustration and self-imposition than girls and this could again be attributed to culture and socialization roles and practices of gender. For instance, right from birth, the male child is portended to be a "bread winner" and people expect him to succeed in life and to be able to take good care of his family i.e. wife, children and other family members. The male child is therefore under constant strain to perform well in school and this is manifested in the form of self-impositions (e.g. getting worried and anxious about writing examinations, zeal to always win etc.). The little disappointment in the form of low exam scores or not being among the best in his class becomes a major source of frustration. Girls on the other hand, based on gender role socialisation, would tend to assume that they have nothing to lose because of the understanding that their future husbands will always take care of them. So being the best in the class does not become an immediate priority. Girls therefore would be less likely to feel self-imposed and get academically frustrated unlike boys.

Pertaining to the socio-economic status of students and their levels of academic frustrations, self-imposition, and academic pressures, those in the high socio-economic group scored the lowest followed by those in the middle class and low status groups respectively on all these three indicators of academic stress. This suggests that the socio-economic status of students is critical in respect of academic stress. The probable reason why there are such socio-economic background differences in academic stress lies in the fact that as students from low socioeconomic backgrounds were more likely to receive low social support, they are self-motivated or inspired to make up for or compensate for their parents' inability to provide their needs [47]. They therefore become selfimposed (e.g. worrying about everything and everybody) with mounting academic pressures (e.g. grade competition) and become frustrated (e.g. as a result of daily hassles) should they encounter any obstacle which hinder their dreams and aspirations. Age and class level did not have significant effects on academic stress and this is consistent with previous studies [43]. The inclusion of age and class level in the analysis may have accounted for the non-significant results observed.

As a limitation in this study, socio-economic status was indexed through parents' highest educational level. Variables such as income and occupation may have some variance but were not indexed in this study. The method used to assess socio-economic status may therefore not 
very accurately represent it. Notwithstanding, the use of parents' education provides as a sense of each student's socio-economic background. The highest education attained by parents was considered because SHS students will have absolutely no idea about their parents' income partly because parents will not disclose their incomes to their children.

Also, perceived social support was measured with reference to family and friends only and did not include support from teachers, church members and others. Expanding the measure of social support to include support from religious members and significant others may help capture a broader view of perceived social support. Notwithstanding, the measurement of perceived social support from family, friends represents the core realistic support that is readily accessible by adolescents. Data were collected from only one SHS so it limits the generalizability of the findings.

\section{Acknowledgements}

Many thanks to Prof. Torbjørn Rundmo for his insight. Thanks to the school and participants who participated in the study. Without funding from the Norwegian Educational Trust (Quota programme) this study would not have been possible, thank you.

\section{REFERENCES}

[1] M. K. Demaray and C. K. Maleck, "The Relationship between Perceived Social Support and Maladjustment for Students at Risk," Psychology in the School, Vol. 39, No. 3, 2002, pp. 305-316.

[2] K. D Lincoln, L. M. Chatters and R. J. Taylor, "Psychological Distress among Black and White Americans: Differential Effects of Social Support, Negative Interaction and Personal Control," Journal of Health and Social Behavior, Vol. 44, No. 3, 2003, pp. 390-407.

[3] I. Kawachi and L. F. Berkman, "Social Ties and Mental Health," Journal of Urban Health: Bulletin of the New York Academy of Medicine, Vol. 78, No. 3, 2001, pp. 458-467.

[4] N. Krause and K. S. Rook, "Negative Interaction in Late Life: Issues in the Stability and Generalizability of Conflict across Relationship," Journal of Gerontology: Psychological Sciences, Vol. 58B, No. 2, 2003, pp. 88-99. http://dx.doi.org/10.1093/geronb/58.2.P88

[5] D. J. Bell, S. L. Foster and E. J. Mash, "Handbook of Behavioral and Emotional Problems in Girls," Kluwer Academic/Plenum Publishers, New York, 2005.

[6] W. R. Lovallo, "Stress and Health: Biological and Psychological Interactions," Sage Publications, Thousand Oaks, 2005.

[7] E. Vaquera and G. Kao, "Do You Like Me as Much as I Like You? Friendship Reciprocity and Its Effects on School Outcomes among Adolescents," Social Science
Research, Vol. 37, No. 1, 2008, pp. 55-72. http://dx.doi.org/10.1016/j.ssresearch.2006.11.002

[8] M. Bolognini, B. Plancherel, W. Bettschart and O. Halfon, "Self-Esteem and Mental Health in Early Adolescence: Development and Gender Differences," Journal of Adolescence, Vol. 19, No. 3, 1996, pp. 233-245. http://dx.doi.org/10.1006/jado.1996.0022

[9] B. Levinger, "Nutrition, Health, and Education for All," United Nations Development Programme, New York, 1994.

[10] B. N. Uchino, "Social Support and Physical Health: Understanding the Health Consequences of Relationships," Yale University Press, New Haven, 2004.

[11] T. Field, M. Diego and C. Sanders, "Adolescents' Parent and Peer Relationship," Adolescence, Vol. 37, No. 145, 2002, pp. 121-130.

[12] B. K. Finch and W. A. Vega, "Acculturation Stress, Social Support, and Self-Rated Health among Latinos in California," Journal of Immigrant Health, Vol. 5, No. 3, 2003, pp. 109-117. http://dx.doi.org/10.1023/A:1023987717921

[13] S. Cohen and T. A. Wills, "Stress, Social Support, and the Buffering Hypothesis," Psychological Bulletin, Vol. 98, No. 2, 1985, pp. 310-357. http://dx.doi.org/10.1037/0033-2909.98.2.310

[14] S. Cohen, "Social Relationships and Health," American Psychologist, Vol. 1, No. 1, 2004, pp. 676-684. http://dx.doi.org/10.1037/0003-066X.59.8.676

[15] B. N. Uchino, J. T. Cacioppo and J. K. Kiecolt-Glaser, "The Relationship between Social Support and Physiological Processes: A Review with Emphasis on Underlying Mechanisms and Implications for Health," Psychological Bulletin, Vo. 119, No. 3, 1996, pp. 488-531. http://dx.doi.org/10.1037/0033-2909.119.3.488

[16] S. Cohen and H. Hoberman, "Positive Events and Social Supports as Buffers of Life Change Stress," Journal of Applied Social Psychology, Vol. 13, No. 2, 1983, pp. 99125.

[17] A. Vaux, "Social support: Theory, Research, and Intervention," Praeger, New York, 1988.

[18] C. Cheng, "Role of Perceived Social Support on Depression in Chinese Adolescents: A Prospective Study Examining the Buffering Model," Journal of Applied Social Psychology, Vol. 27, No. 9, 1997, pp. 800-820. http://dx.doi.org/10.1111/j.1559-1816.1997.tb00660.x

[19] R. W. Landow and D. S. Glenwick, "Stress and Coping in Homeless Children," Journal of Social Distress and the Homeless, Vol. 8, No. 2, 1999, pp. 79-93. http://dx.doi.org/10.1023/A:1021394610436

[20] I. Kawachi and L. F. Berkman, "Social Cohesion, Social Capital, and Health," In: L. F. Berman and I. Kawachi, Eds., Social Epidemiology, Oxford University Press, New York, 2000, pp. 174-190.

[21] G. E. Capowich, P. Mazerolle and A. Piquero, "General Strain Theory, Situational Anger, and Social Networks: An Assessment of Conditioning Influences," Journal of Criminal Justice, Vol. 29, No. 5, 2001, pp. 445-461. http://dx.doi.org/10.1016/S0047-2352(01)00101-5 
[22] A. Baum, T. A. Revenson and J. E. Singer, Ed., "Handbook of Health Psychology," Lawrence Erlbaum Associates, New Jersey, 2001.

[23] S. Cohen, "Psychosocial Models of Social Support in the Etiology of Physical Disease," Health Psychology, Vol. 7, No. 3, 1988, pp. 269-297. http://dx.doi.org/10.1037/0278-6133.7.3.269

[24] L. F. Berkman, "The Role of Social Relations in Health Promotion," Psychosomatic Medicine, Vol. 57, No. 3, 1995, pp. 245-254.

[25] L. F. Berkman, T. Glass, I. Brissette and T. E. Seeman, "From Social Integration to Health: Durkheim in the New Millennium," Social Science and Medicine, Vol. 51, No. 6, 2000, pp. 843-857. http://dx.doi.org/10.1016/S0277-9536(00)00065-4

[26] E. Delistamati, M. A. Samakouri, E. A. Davis, T. Vorvolakos, K. Xenitidis and M. Livaditis, "Interpersonal Support Evaluation List (ISEL) —College Version: Validation and Application in a Greek Sample," International Journal of Social Psychiatry, Vol. 52, No. 6, 2006, pp. $552-560$.

[27] N. Lin, X. Ye and W. M. Ensel, "Social Support and Depressed Mood: A Structural Analysis," Journal of Health and Social Behavior, Vol. 40, No. 4, 1999, pp. 344-359.

[28] T. A. Wills, G. McNamara D. Vaccaro, "Parental Education Related to Adolescent Stress-Coping and Substance Use: Development of a Mediational Model," Health Psychology, Vol. 14, No. 5, 1995, pp. 464-478. http://dx.doi.org/10.1037/0278-6133.14.5.464

[29] R. D. Conger, K. J. Conger, G. H. Elder, F. O. Lorenz, R. L. Simons and L. B. Whitbeck, "Family Economic Stress and Adjustment of Early Adolescent Girls," Developmental Psychology, Vol. 29, No. 2, 1993, pp. 206-219. http://dx.doi.org/10.1037/0012-1649.29.2.206

[30] A. Gecková, J. P. Van Dijk, R. Stewart, J. W. Groothoff and D. Post, "Influence of Social Support on Health among Gender and Socio-Economic Groups of Adolescents," European Journal of Public Health, Vol. 13, No. 1, 2003, pp. 44-50.

http://dx.doi.org/10.1093/eurpub/13.1.44

[31] B. M. Gadzella, "Student-Life Stress Inventory: Identification of and Reactions to Stressors," Psychological Reports, Vol. 74, No. 2, 1994, pp. 395-402. http://dx.doi.org/10.2466/pr0.1994.74.2.395

[32] D. P. Goldberg, "The Detection of Psychiatric Illness by Questionnaire," Oxford University Press, Oxford, 1972.

[33] M. Procidano and K. Heller, "Measures of Perceived Social Support from Friends and from Family: Three Validation Studies," American Journal of Community Psychology, Vol. 11, No. 1, 1983, pp. 1-24. http://dx.doi.org/10.1007/BF00898416

[34] S. Henderson, "Social Relationships, Adversity, and Neurosis: An Analysis of Prospective Observations," British Journal of Psychiatry, Vol. 138, No. 1, 1981, pp. 391398.
[35] M. A. Hoffman, V. Ushpiz and R. Levy-Shiff, "Social Support and Self-Esteem in Adolescence," Journal of Youth and Adolescence, Vol. 17, No. 4, 1988, pp. 307316.

[36] H. B. Kaplan, C. Robbins and S. S. Martin, "Antecedents of Psychological Distress in Young Adults: Self-Rejection, Deprivation of Social Support, and Life Events," Journal of Health and Social Behavior, Vol. 24, No. 3, 1983, pp. 230-244.

[37] H. C. Huang, K. K. Hwang and Y. H. Ko, "Life Stress, Attribution Style, Social Support, and Depression among University Students," Acta Psychologica Taiwanica, Vol. 25, No. 1, 1983, pp. 31-47.

[38] R. S. Lazarus and S. Folkman, "Stress, Appraisal, and Coping," Springer, New York, 1984.

[39] R. A. Thoits, "Social Support as Coping Assistance," Journal of Consulting and Clinical Psychology, Vol. 54, No. 4, 1986, pp. 416-423. http://dx.doi.org/10.1037/0022-006X.54.4.416

[40] B. L. Wilcox, "Social Support, Life Stress, and PsychoLogical Adjustment: A Test of the Buffering Hypothesis," American Journal of Community Psychology, Vol. 9, No. 4, 1981, pp. 371-386.

[41] V. M. Simonds and V. E. Whiffen, "Are Gender Differences in Depression Explained by Gender Differences in Co-Morbid Anxiety?" Journal of Affective Disorders, Vol. 77, No. 3, 2003, pp. 197-202. http://dx.doi.org/10.1016/S0165-0327(02)00113-1

[42] B. Piko, "Social Support and Health in Adolescence: A Factor Analytical Study," British Journal of Health Psychology, Vol. 3, No. 4, 1998, pp. 33-44. http://dx.doi.org/10.1111/j.2044-8287.1998.tb00578.x

[43] O. Olsen, L. Iversen and S. Sabore, "Age and the Operationalization of Social Support," Social Science and Medicine, Vol. 32, No. 7, 1991, pp. 767-771. http://dx.doi.org/10.1016/0277-9536(91)90302-S

[44] R. J. Turner, B. Wheaton and D. A. Lloyd, "The Epidemiology of Social Stress," American Sociological Review, Vol. 60, No. 1, 1995, pp. 104-125. http://dx.doi.org/10.2307/2096348

[45] N. E. Mahon, A. Yarcheski and T. J. Yarcheski, "Differences in Social Support and Loneliness in Adolescents According to Developmental Stage and Gender," Public Health Nursing, Vol. 11, No. 5, 1994, pp. 361-368. http://dx.doi.org/10.1111/j.1525-1446.1994.tb00199.x

[46] J. G. Grzywacz, D. M. Almeida, S. D. Neupert and S. L. Ettner, "Socioeconomic Status and Health: A MicroLevel Analysis of Exposure and Vulnerability to Daily Stressors," Journal of Health and Social Behavior, Vol. 45, No. 1, 2004, pp. 1-16.

[47] H. Brutsaert and M. Van Houtte, "Gender Context of Schooling and Levels of Stress among Early Adolescent Pupils," Education and Urban Society, Vol. 37, No. 1, 2004, pp. 58-73. 